\title{
Valoración de las herramientas y metodologías activas en el Grado en Ingeniería de Obras Públicas
}

Tatiana García-Segura ${ }^{a}$, José V. Martít y Víctor Yepes ${ }^{\mathrm{c}}$

aICITECH. Universitat Politècnica de València, tagarse@cam.upv.es, ' Innovación y Calidad Educativa EXCELCON. Departamento de Ingeniería de la Construcción y Proyectos de Ingeniería Civil. Universitat Politècnica de València, jvmartia@cst.upv.es, y 'ICITECH. Equipo de Innovación y Calidad Educativa EXCELCON. Departamento de Ingeniería de la Construcción y Proyectos de Ingeniería Civil. Universitat Politènnica de València, vyepesp@cst.upv.es.

\begin{abstract}
The change in the orientation of the higher education system has given rise to a teaching model focuses on student learning and skills acquisition. The communication presents the tools used for the "inverse class" teaching. A questionnaire has been designed to evaluate the active methodology and tools used in the class. Results indicate that the most valued tool is Lessons, followed by PoliformaT resources and pdf slides. Polimedia videos and videos of constructive methods present little deviation, indicating that all students agree with the usefulness of such technologies. It is also important to note that there is no disagreement with the active methodology. In particular, the correction of questions is the most valued activity in the learning process.
\end{abstract}

Keywords: technological resources, tools, active methodology, inverse class, questionnaire

\footnotetext{
Resumen

El cambio en la orientación del sistema de educación superior ha dado lugar a un modelo de enseñanza centrada en el aprendizaje del estudiante y la adquisición de habilidades. La comunicación presenta la valoración por parte de los alumnos de los recursos utilizados en la docencia de la "clase inversa". Se ha diseñado un cuestionario para evaluar la metodología activa $y$ herramientas utilizadas. De los resultados se destaca que la herramienta mejor valorada es Lessons, seguida de Recursos de Poliformat y diapositivas en pdf. Los vídeos de polimedia y los vídeos de procedimientos constructivos presentan poca desviación, indicando que todos los alumnos están de acuerdo con la utilidad de dichas tecnologías. También es importante destacar que no hay ningún alumno en desacuerdo con la metodología activa. En concreto, la corrección de entregables es la actividad más valorada en el proceso del aprendizaje.
}

Palabras clave: recursos tecnológicos, herramientas, metodología activa, clase inversa, cuestionario 


\section{Introducción}

En los últimos años, ha habido un giro significativo en la orientación del sistema de educación superior como consecuencia de la Declaración de Bolonia (1999). El Espacio Europeo de Educación Superior (EEES) pretende construir un área de acuerdos y procesos comunes. Las universidades han adaptado sus estudios para cumplir con el compromiso. En primer lugar, se ha modificado la estructura de la enseñanza universitaria en España, dando lugar a los estudios de Grado, Máster y Doctorado, basados en los sistemas de transferencia de créditos europeos (ECTS). En segundo lugar, se ha revisado el modelo de enseñanza para adecuarlo a una formación centrada en el aprendizaje del estudiante y la adquisición de habilidades. En este sentido, la propuesta curricular debe seguir la nueva filosofía pedagógica, es decir, se centra en la adquisición de competencias en lugar de en la adquisición de conocimientos. Además, se promueve la competitividad, empleabilidad y movilidad.

Se distinguen dos categorías principales de concepción pedagógica (Kember et al. 2001): la enseñanza como transmisión del conocimiento y la enseñanza como ayuda al aprendizaje. La primera considera a los estudiantes como receptores pasivos de información. Sin embargo, la segunda concepción pedagógica se centra en fomentar el aprendizaje de los estudiantes. En este último caso, se busca que los estudiantes asuman un papel activo y participativo en el proceso educativo (Kane 2004). A pesar de que la profesión de ingeniero está ligada al análisis y resolución de problemas, en los estudios de ingeniería se ha tendido hacia la enseñanza deductiva. Para acabar con esta tendencia, la Universitat Politècnica de València (UPV), y en particular la Escuela Técnica Superior de Ingenieros de Caminos, Canales y Puertos, está impulsando el aprendizaje por competencias para conseguir un perfil académico-profesional más enfocado a las demandas actuales y futuras. En este contexto se encuentra el fomento de metodologías como la "clase inversa", en la que el profesor planifica actividades de aprendizaje para que el alumno sea el protagonista de su aprendizaje, participe en la formación de su propio conocimiento y se les permita la autoevaluación.

El Equipo de Innovación y Calidad Educativa EXCELCON, centrado en la gestión de proyectos y construcción (Pellicer et al. 2014; Pellicer et al. 2017), destaca la importancia de habilidades como liderazgo, gestión, comunicación, trabajo en equipo y pensamiento crítico, para puestos de gestión en la industria de la construcción (Yepes at al. 2012). En este sentido, se ha propuesto una serie de requerimientos que deben cubrir los estudios de posgrado en el ámbito de la gestión de la construcción (Yepes at al. 2016), así como una metodología innovadora para planificar los estudios de máster (Pellicer et al. 2013), en consonancia con un modelo que evalúa la desviación de cada programa a las necesidades de mercado (Yepes at al. 2012). Las competencias transversales, como es el caso del pensamiento crítico, pueden desarrollarse en las aulas a la vez que se trabajan otras competencias técnicas (García-Segura et al. 2016; Yepes et al. 2016). Asimismo, se pueden utilizar metodologías activas como herramientas para concienciar a los alumnos de los nuevos retos que presenta la sostenibilidad (Pellicer et al. 2016; Penadés-Plà et al. 2016; García-Segura et al. 2015). Este estudio analiza las herramientas y la metodología activa en 
estudios de ingeniería, particularmente en la asignatura Tipologías y Procedimientos de las Construcciones de la Ingeniería Civil (I).

\section{Objetivos}

Esta comunicación tiene como objetivos los que se relacionan a continuación:

1. Mostrar los recursos tecnológicos y herramientas utilizados en la asignatura Tipologías y Procedimientos de las Construcciones de la Ingeniería Civil (I).

2. Valorar la importancia de las herramientas y metodologías activas en el proceso de aprendizaje.

\section{Desarrollo de la innovación}

La innovación planteada consiste en la utilización de herramientas, así como metodologías activas para facilitar el proceso de enseñanza-aprendizaje. Este estudio analiza las respuestas de los estudiantes a un cuestionario diseñado para evaluar la utilización de herramientas y metodología de "clase inversa" en la asignatura Tipologías y Procedimientos de las Construcciones de la Ingeniería Civil (I) durante el curso académico 2016-2017. Esta asignatura pertenece al segundo año del Grado en Ingeniería de Obras Públicas.

Las variables estudiadas se encuentran relacionadas con las características del alumno, las notas de los exámenes y su actitud frente a las tecnologías educativas y metodologías activas de aprendizaje. Se utiliza un cuestionario de preguntas de respuesta múltiple para conocer las características del estudiante y las notas, mientras su actitud frente a la innovación planteada se evalúa mediante la escala de Likert (1-5), siendo su valoración creciente desde 1 hasta 5. El valor "1" significa para el alumno, que se podría prescindir de la herramienta, mientras que el "5" equivale a considerar dicha herramienta como imprescindible. A continuación se muestra las variables y las opciones planteadas en el cuestionario:

- $\quad$ Sexo: 1(Hombre), 2(Mujer)

- Edad: 1(18-19), 2(20-21), 3(más de 21 años),

- Horas de estudio semanales: 1(< $1 \mathrm{~h} /$ semana), 2(1-3h/semana), 3(>3 h/semana)

- Notas del primer parcial: 1(no presentado), 2(suspenso), 3(aprobado), 4(notable), 5(sobresaliente)

- Notas segundo parcial: 1(no presentado), 2(suspenso), 3(aprobado), 4(notable), 5(sobresaliente)

- Actitud frente a la utilidad de la herramienta recursos de PoliformaT: 1,2,3,4,5

- Actitud frente a la utilidad de las diapositivas en pdf : 1,2,3,4,5

- Actitud frente a la utilidad de las diapositivas en Power Point: 1,2,3,4,5

- Actitud frente a la utilidad de los vídeos Polimedia: 1,2,3,4,5

- $\quad$ Actitud frente a la utilidad del libro de apuntes: $1,2,3,4,5$

(c) ) EY-NC-ND 2017, Universitat Politècnica de València 
- Actitud frente a la utilidad de los vídeos de procedimientos constructivos: $1,2,3,4,5$

- Actitud frente a la utilidad de la herramienta Lessons de PoliformaT: 1,2,3,4,5

- Actitud frente a la utilidad del Portafolio en espacio compartido del PoliformaT: $1,2,3,4,5$

- Actitud frente a la utilidad de la metodología de la clase inversa: $1,2,3,4,5$

- Actitud frente a la utilidad de la corrección de entregables: $1,2,3,4,5$

\section{Herramienta "Recursos" de PoliformaT}

La carpeta "Recursos" contiene documentos útiles para el desarrollo de la asignatura y el aprendizaje del alumno. No sólo se proporcionan los temas que se ven en clase, también se facilita bibliografía para que los alumnos puedan ampliar sus conocimientos.

\section{Diapositivas en pdf y Power Point}

Las diapositivas facilitadas a los alumnos muestran los contenidos de la asignatura distribuidos por temas. Éstas aportan imágenes y ejemplos que no se recogen en el libro de la asignatura. Las diapositivas se facilitan en pfd y Power Point. Las diapositivas en Power Point disponen de enlaces a vídeos. Se analiza si los alumnos prefieren las diapositivas en Power Point o si se puede prescindir de éstas.

\section{$\underline{\text { Vídeos Polimedia }}$}

Es una tecnología educativa basada en vídeos para presentar un tema. El profesor explica los contenidos con la ayuda de diapositivas. Además, el profesor aparece en el vídeo para mejorar la comunicación no verbal. Esta tecnología educativa permite que el alumno pare la explicación del profesor cuando necesite más tiempo para tomar nota o consultar algo.

\section{Libro de apuntes}

El libro de la asignatura contiene los contenidos explicados en detalle. Los alumnos pueden recurrir al libro para entender mejor el tema. Además, éste contiene bibliografía de consulta.

\section{Vídeos de procedimientos constructivos}

El objetivo de la asignatura Tipologías y Procedimientos de las Construcciones de la Ingeniería Civil (I) es que los alumnos aprendan diferentes procedimientos constructivos. Es por ello que se consideró importante aportar videos de procedimientos constructivos correspondientes a la ingeniería civil.

\section{Lessons}

Esta herramienta permite conocer la planificación completa de las clases. El profesor crea itinerarios formativos para que los alumnos dispongan de todos los elementos necesarios para el desarrollo de las clases. Los alumnos conocen de antemano los temas que se verán en cada clase, los documentos que necesitarán, así como las actividades previas y de aula que deberán realizar. Se crean condiciones de acceso a los distintos elementos publicados. Esta herramienta es propia de las metodologías activas como puede ser la metodología de clase inversa. 


\section{Portafolio}

El portafolio es un conjunto documental que recoge las tareas realizadas por el alumno durante el curso. Los estudiantes comparten con el profesor un espacio virtual donde se incluyen los documentos que evidencian las habilidades desarrolladas y los conocimientos adquiridos. Esta herramienta permite a su vez realizar el proceso de evaluación, ya que muestra las habilidades, esfuerzo, progreso y logros de los estudiantes.

\section{Clase inversa}

Este tipo de clase se sale del modelo de enseñanza tradicional, pues la clase magistral se sustituye por "deberes" en el aula, mientras los contenidos son aprendidos online fuera del aula. En esta asignatura, se ha decidido seguir el modelo de clase inversa incluyendo la lección magistral participativa. Los contenidos son presentados mediante distintas herramientas (diapositivas, vídeos, libros, otros documentos de PoliformaT...). Durante las clases, se diseñan distintas actividades de aprendizaje para profundizar en los contenidos y ofrecerles feedback. Asimismo, se utilizarán estas actividades para que el alumno adquiera competencias transversales. Como proceso de evaluación del aprendizaje, se recomienda la evaluación formativa. Esta metodología es consistente con la taxonomía Bloom, ya que deja los niveles inferiores (recordar, entender) para el desarrollo en casa, y los superiores (aplicar, analizar, evaluar y crear) para desarrollarlos en el aula. Asimismo, se sigue la estrategia del EEES, ya que el alumno será un "ser activo", mientras el profesor hará de "guía" en el proceso de aprendizaje.

\section{Corrección de entregables}

Para llevar a cabo un aprendizaje activo, los alumnos realizan entregables previos al desarrollo de cada clase. Asimismo, en clase también se realizan entregables con la supervisión de los profesores. Las respuestas se pueden resolver con una lectura atenta del libro correspondiente y demás recursos de PoliformaT. Además, los alumnos pueden consultar distintas fuentes como la bibliografía de la asignatura, internet, etc. Durante el desarrollo de las clases, se corrigen los entregables mediante una evaluación formativa. El profesor aprovecha para matizar, ampliar y exponer la materia.

\section{Resultados}

A continuación se analizan los resultados obtenidos del cuestionario. El tamaño de la muestra ha sido $N=22$. De acuerdo con la Figura 1, el $73 \%$ de los estudiantes es hombre, mientras el $27 \%$ es mujer. El $73 \%$ de los estudiantes tiene más de 21 años, el $41 \%$ tiene entre 20 y 21 años y el 5\% tiene menos de 20 años. Las horas dedicadas al estudio semanal fueron menos de 1 hora (18\%), entre 1 y 3 horas (64\%) y más de 3 horas (18\%). Las notas del primer parcial fluctúan entre no presentado y sobresaliente. El mayor porcentaje corresponde notable (32\%), seguido de aprobado (23\%), sobresaliente (23\%), suspenso (18\%) y no presentado (5\%). Sin embargo, todos los encuestados se presentaron al segundo parcial y ninguno obtuvo la nota de sobresaliente. El 27\% de los estudiantes suspendió el examen, mientras el $50 \%$ obtuvo aprobado y el $23 \%$ notable. 


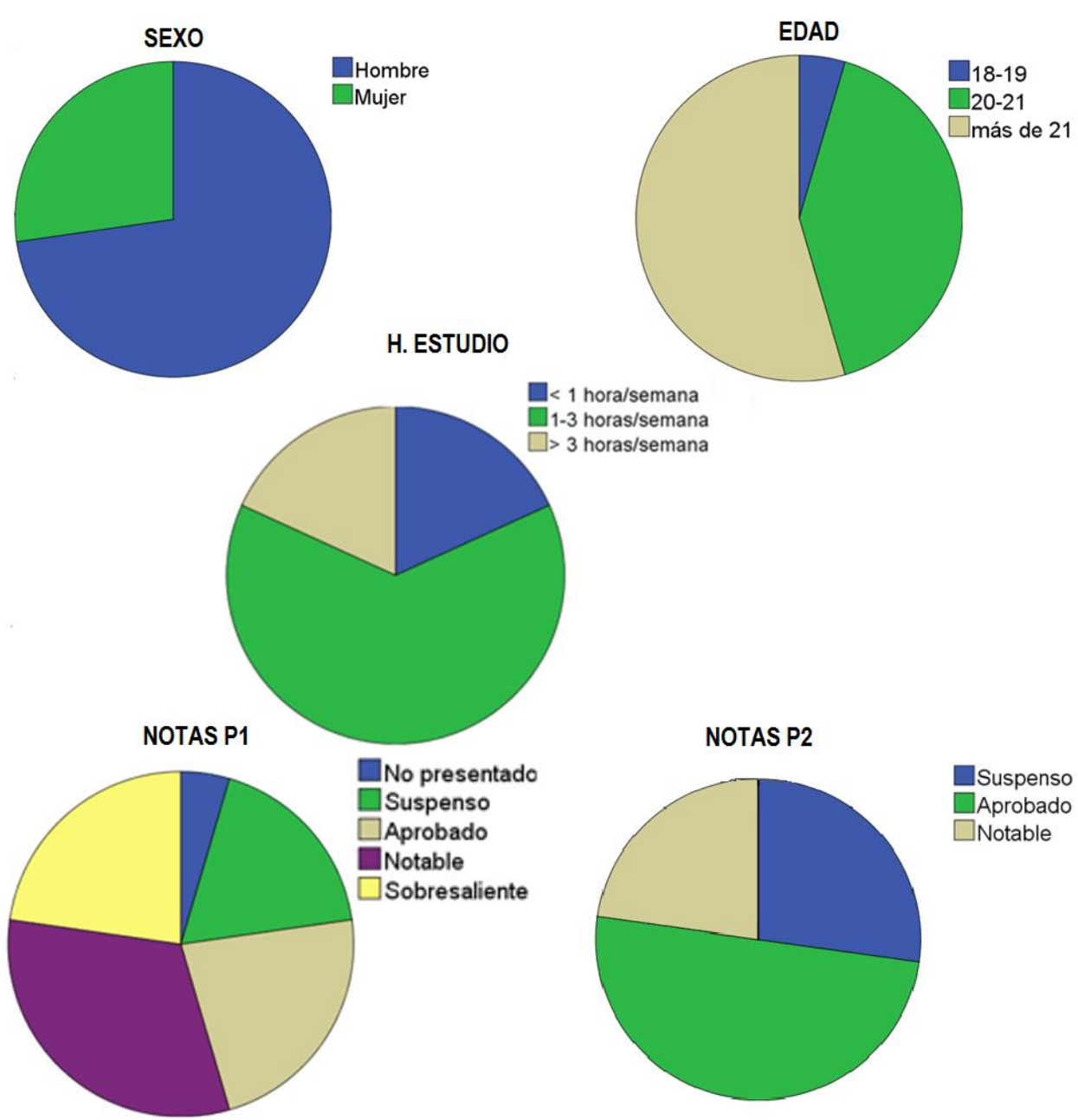

Fig. 1 Caracterización de la muestra analizada

La Tabla 1 resume los resultados estadísticos de las variables. Se agrupan las respuestas en calificaciones negativas (1,2), neutras (3) y positivas (4,5). La Tabla 2 muestra los porcentajes de cada grupo de respuestas. Respecto a las tecnologías educativas, los estudiantes están generalmente de acuerdo con la utilización de la herramienta recursos de PoliformaT. Ningún estudiante está en desacuerdo con esta herramienta. Las diapositivas las prefieren generalmente en pdf $(86 \%)$. Por tanto, los resultados indican que se podría prescindir de las diapositivas en Power Point. Hay dos alumnos muy en desacuerdo con las diapositivas en Power Point y cuatro en desacuerdo. Esto representa el 27\% de la muestra. Respecto a las diapositivas en pdf, no hay ningún estudiante muy en desacuerdo, y sólo dos en desacuerdo. La distribución de opiniones referentes a las diapositivas en pdf queda del modo siguiente: negativas $(9 \%)$, neutras $(5 \%)$ y positivas $(86 \%)$.

Los videos de polimedia están muy bien valorados por los estudiantes, pues ninguno está en desacuerdo con esta tecnología y el 55\% muestra una actitud positiva. El libro de apuntes

(c)) BY-NC-ND 2017, Universitat Politècnica de València 
tiene peor puntación, el 50\% de las calificaciones han sido negativas. Esto indica que los estudiantes prefieren que el profesor les explique el tema, a tener que leerlo ellos en un libro. Por tanto, la tecnología educativa de los videos de Polimedia funciona adecuadamente. El $45 \%$ de los estudiantes muestra una actitud neutra, mientras el $55 \%$ de los estudiantes muestra una actitud positiva. Los vídeos de procedimientos constructivos también son muy bien valorados. Ningún estudiante está en desacuerdo, mientras el $73 \%$ de los estudiantes está de acuerdo con esta tecnología.

La herramienta Lessons es la mejor valorada de todas, y la que menos desviación presenta. El 59\% de los estudiantes están de acuerdo, y el $41 \%$ está muy de acuerdo con esta herramienta. La opinión respecto al Portafolio presenta mucha divergencia. Mientras hay 2 estudiantes muy en desacuerdo con esta herramienta, 7 estudiantes están muy de acuerdo.

En relación a las metodologías activas, no hay ningún alumno en desacuerdo. El 14\% tiene una actitud neutra y el $86 \%$ tiene una actitud positiva hacia la metodología de la clase inversa. Asimismo, la corrección de entregables, también está muy bien valorada. Ésta presenta mejor media y menor desviación típica que la metodología de clase inversa en general. El 5\% de las calificaciones han sido neutras y el $95 \%$ han sido positivas. Por tanto, hay alumnos que prefieren la corrección de entregables a otras actividades de la metodología activa.

Tabla 1. Descripción estadística de las variables

\begin{tabular}{ccccc}
\hline & Media & Desviación típica & Mínimo & Máximo \\
\hline Recursos PoliformaT & 4,32 & 0,72 & 3 & 5 \\
Diapositivas en pdf & 4,14 & 0,89 & 2 & 5 \\
Diapositivas en Power Point & 3,32 & 1,25 & 1 & 5 \\
Vídeos Polimedia & 3,82 & 0,85 & 3 & 5 \\
Libro de apuntes & 2,95 & 1,21 & 1 & 5 \\
Vídeos procedimientos constructivos & 3,95 & 0,72 & 3 & 5 \\
Lessons & 4,41 & 0,50 & 4 & 5 \\
Portafolio & 3,64 & 1,33 & 1 & 5 \\
Metodología de la clase inversa & 4,23 & 0,69 & 3 & 5 \\
Corrección entregables & 4,68 & 0,57 & 3 & 5
\end{tabular}


Tabla 2. Porcentajes de calificaciones

\begin{tabular}{cccc}
\hline & $\begin{array}{c}\text { Porcentaje de } \\
\text { calificaciones } \\
\text { negativas (1,2) }\end{array}$ & $\begin{array}{c}\text { Porcentaje de } \\
\text { calificaciones } \\
\text { neutras (3) }\end{array}$ & $\begin{array}{c}\text { Porcentaje de } \\
\text { calificaciones } \\
\text { positivas (4,5) }\end{array}$ \\
\hline Recursos PoliformaT & $0 \%$ & $14 \%$ & $86 \%$ \\
Diapositivas en pdf & $9 \%$ & $5 \%$ & $86 \%$ \\
Diapositivas en Power Point & $27 \%$ & $23 \%$ & $50 \%$ \\
Vídeos Polimedia & $0 \%$ & $45 \%$ & $55 \%$ \\
Libro de apuntes & $50 \%$ & $14 \%$ & $36 \%$ \\
Vídeos procedimientos constructivos & $0 \%$ & $27 \%$ & $73 \%$ \\
Lessons & $0 \%$ & $0 \%$ & $100 \%$ \\
Portafolio & $23 \%$ & $14 \%$ & $64 \%$ \\
Metodología de la clase inversa & $0 \%$ & $14 \%$ & $86 \%$ \\
Corrección entregables & $0 \%$ & $5 \%$ & $95 \%$ \\
\hline
\end{tabular}

\section{Conclusiones}

La presente comunicación presenta la metodología utilizada para llevar a cabo el proceso de enseñanza-aprendizaje en la asignatura Tipologías y Procedimientos de las Construcciones de la Ingeniería Civil (I). Se muestran tanto las estrategias de aprendizaje activo como las herramientas y tecnologías educativas utilizadas. Se analizan las respuestas de los estudiantes a un cuestionario. Las variables estudiadas están relacionadas con el perfil de los estudiantes y su actitud frente a la utilidad de la herramienta recursos de PoliformaT, diapositivas en pdf, diapositivas en Power Point, vídeos Polimedia, libro de apuntes, vídeos de procedimientos constructivos, herramienta Lessons de PoliformaT, metodología de la clase inversa y corrección de entregables.

Los estudiantes están generalmente de acuerdo con la utilización de la herramienta recursos de PoliformaT. Prefieren las diapositivas en pdf que en Power Point. Los videos de polimedia y los vídeos de procedimientos constructivos están muy bien valorados por los estudiantes. Ningún estudiante está en desacuerdo con esta tecnología. Les resulta más útil la explicación on-line de los contenidos, que el libro de apuntes. La herramienta Lessons es la mejor valorada de todas, y la que menos desviación presenta. Respecto a la metodología activa, no hay ningún estudiante en desacuerdo. La corrección de entregables, en particular, es la más "imprescindible". De los resultados se deduce que hay alumnos que prefieren la corrección de entregables a otras actividades de la metodología activa.

\section{Agradecimientos}

Los autores agradecen el apoyo recibido por la Universitat Politècnica de València (Equipo de Innovación y Calidad Educativa EXCELCON). 


\section{Referencias}

BOLOGNA DECLARATION (1999). "Towards the European Higher European Area". En: Conference of Ministers responsible for Higher Education in 29 European countries. June, Bologna, Italy.

GARCÍA-SEGURA, T. et al. (2015). "Hybrid harmony search for sustainable design of posttensioned concrete box-girder pedestrian bridges", en Engineering Structures, vol 92, p.112-122.

GARCÍA-SEGURA, T. et al. (2016). "Assessment of transverse and specific competences in civil engineering studies: 'critical thinking"'. En: 11th International Technology, Education and Development Conference (INTED 2017). 6-8 de marzo, Valencia, pp. 3683-3692.

KANE, L. (2004). "Educators, learners and active learning methodologies", en International Journal of Lifelong Education, vol. 23, issue 3, p. 275-286.

KEMBER, D., KWAN, K.P. y LEDESMA, J. (2001). "Conceptions of good teaching and how they influence the way adults and school leavers are taught", en International Journal of Lifelong Education, vol. 20, issue 5, p. 393-404.

PELLICER, E., SIERRA, L.A. y YEPES, V. (2016). "Appraisal of infrastructure sustainability by graduate students using an active-learning method“, en Journal of Cleaner Production, vol. 113, p. 884-896.

PELLICER, E., YEPES, V. y ORTEGA, A.J. (2013). "Method for planning a graduate program in construction management", en Journal of Professional Issues in Engineering Education and Practice $A S C E$, vol 139, issue 1,p. 33-41.

PELLICER, E., et al. (2017). "Market demands on construction management: A view from graduate students", en Journal of Professional Issues in Engineering Education and Practice ASCE, (accepted, in press).

PELLICER, E. et al. (2014). Construction Management. New York: Wiley Blackwell.

PENADÉS-PLÀ, V. et al. (2016). "A review of multi-criteria decision making methods applied to the sustainable bridge design”, en Sustainability, vol. 8, issue 12, p. 1295.

YEPES, V., PELLICER, E. y ORTEGA, J.A. (2012). "Designing a benchmark indicator for managerial competences in construction at the graduate level", en Journal of Professional Issues in Engineering Education and Practice ASCE, vol. 138, issue 1, p. 48-54

YEPES, V. et al. (2016). "Acquisition of competences in a Master Degree in Construction Management". En: 10th International Technology, Education and Development Conference (INTED 2016). 7-9 de marzo, Valencia, pp. 718-727.

YEPES, V., MARTI, J.V. y GARCÍA-SEGURA, T. (2016). "Desarrollo y evaluación de la competencia transversal "pensamiento crítico" en el grado de ingeniería civil". En: Jornadas de Innovación Educativa y Docencia en Red IN-RED 2016. 7-8 de julio, Valencia, pp. 1-14. 